\title{
Neurophysiological features of verbal divergent thinking in elderly scientists
}

\author{
E. Privodnova ${ }^{1 *}$, N. Volf ${ }^{1,2}$ \\ ${ }^{1}$ Scientific Research Institute of Physiology and Basic Medicine, Novosibirsk, Russia \\ ${ }^{2}$ Novosibirsk State University, Novosibirsk, Russia \\ *e-mail: privodnovaeu@physiol.ru
}

Key words: alpha, divergent thinking, elderly, interhemispheric asymmetry, verbal memory

Motivation and Aim: Cognitive training and rich information environment can contribute to high productivity of divergent problem solving in aging via specific reorganization of brain activity. We investigated scientific activity and originality-related differences in EEG activity during verbal divergent problem solving. Alpha power varies as a function of originality of ideas and creativity-related task demands [1], so, we have considered alpha1, 2 frequency bands. Since alpha synchronization is associated with access to long-term memory [2], we explored its relation with parameters of verbal memory.

Methods and Algorithms: Elderly adults participated in the study: 43 were engaged in professional scientific activity $(\mathrm{SA}, \mathrm{M}=65.2$ years, $\mathrm{SE}=0.97)$ and 41 were continuing their professional non-scientific activities (non-SA, $\mathrm{M}=62.4, \mathrm{SE}=0.99$ ). EEG data were recorded from 52 electrodes during divergent test "Alternate Uses Task" implementation. Alpha 1, 2 power was calculated using FFT; power reactivity was calculated as $\log$ (test)$\log$ (prestimulus). Verbal memory was assessed by dichotic test. ANOVA, regression and correlation analysis were used.

Results: SA outperformed non-SA elderly in originality and amount of ideas, speed of divergent problem solving. Interhemispheric asymmetry of alpha power reactivity with larger values in the right then in the left hemisphere was significant in low original SA subjects only ( $p=0.008)$. For all subjects, dominance of right lateralized verbal memory (over left lateralized one) made positive impact in that interhemispheric alpha power asymmetry $(p<0.004)$. High number of successfully retrieved words presented in the right hemisphere was accompanied by lower originality of creative ideas in SA subjects only $(r=-0.46, p=0.003)$.

Conclusion: Interhemispheric alpha power reactivity asymmetry with larger values in the right then in the left hemisphere during verbal divergent problem solving is associated with the predominance of right hemispheric strategies of verbal memory. This effect is related to low creative originality in SA elderly. The possibility that different information processing strategies contribute to creative originality in SA and non-SA elderly is discussed.

Acknowledgements: The reported study was funded by RFBR and Government of the Novosibirsk region according to the research project № 17-46-540705.

\section{References}

1. Fink A., Benedek M. (2014) EEG alpha power and creative ideation. Neurosci Biobehav Rev. 44: 111-123.

2. Klimesch W. (2012) Alpha-band oscillations, attention, and controlled access to stored information. Trends CognSci 16(12):606-617. 